\title{
A Rare Cause of Acute Colonic Pseudo-obstruction: Ogilvie's Syndrome Caused by Herpes Zoster
}

TO THE EDITOR: Acute colonic pseudo-obstruction (ACPO, also called Ogilvie's syndrome) is a rare disorder characterized by colonic dilatation in the absence of a mechanical cause. ${ }^{1}$ Although $\mathrm{ACPO}$ is an important cause of morbidity and mortality, the pathophysiology is not completely understood. Predisposing factors for ACPO are non-operative trauma, infection (pneumonia and sepsis), cardiac disease (myocardial infarction and heart failure), obstetric/gynecological abdominal/pelvic surgery, neurological disorders (Parkinson's disease, spinal cord injury, multiple sclerosis, and Alzheimer's disease), orthopedic surgery, and various other medical and surgical conditions. ${ }^{2}$

We recently experienced a rare case of ACPO caused by herpes zoster. A 75-year-old female was admitted for right back pain for 4 days. She also complained of a sudden onset of constipation with abdominal distension and discomfort. Physical examination revealed erythematous vesicles involving the right L4 dermatome and marked abdominal distension without tenderness (Fig. 1). Laboratory test results showed no overwhelming abnormalities except sodium 132 (normal: 136-147) mEq/L, chloride 97 (normal: 98-110) $\mathrm{mEq} / \mathrm{L}$, and C-reactive protein 1.76 (normal: 0.0-0.3) $\mathrm{mg} / \mathrm{dL}$. Abdominal radiography showed diffuse colonic dilatation (Fig. 2A and 2B), however abdominopelvic computed tomography revealed no definite obstructive lesion in the colon. ACPO associated with herpes zoster was diagnosed. There was no underlying disease that could cause ACPO. She was treated with intravenous fluids, oral valaciclovir, bisacodyl, and polyethylene glycol. After 3 days, she had a small bowel movement with gas passing. However, colonic dilatation was still present. Her bowel symptoms gradually improved after conservative management and the colonic dilatation disappeared after 10 days (Fig. 2C and 2D).

Herpes zoster mainly involves the sensory neurons. However, motor nerve involvement occurs in about $5 \%$ of cases, resulting in diaphragmatic and bladder paralysis. ${ }^{3}$ Unilateral abdominal wall paralysis due to motor nerve involvement by herpes has rarely been reported. ${ }^{4}$ Gastrointestinal manifestations of herpes zoster such as colonic pseudo-obstruction and gastroparesis are extremely rare. The mechanism of colonic pseudo-obstruction in herpes zoster is not known. However, extrinsic autonomic or motor neuron involvement has been suggested. ${ }^{5}$ In addition, direct invasion of the intestinal muscularis propria and myenteric plexus by varicellazoster virus was reported in an HIV-positive patient with Burkitt lymphoma. ${ }^{5}$ The interval between onset of the herpes zoster rash and bowel symptom in patients with zoster-associated ACPO is variable. A literature review reported that rashes developed 1 day to several weeks after bowel symptom onset in 48\%, 2 days to 1 month before bowel symptom onset in $28 \%$, and simultaneously in $24 \%$.

Zoster is not uncommon and is generally self-limited. However, it is a rare cause of ACPO, which has a high mortality rate if isch-

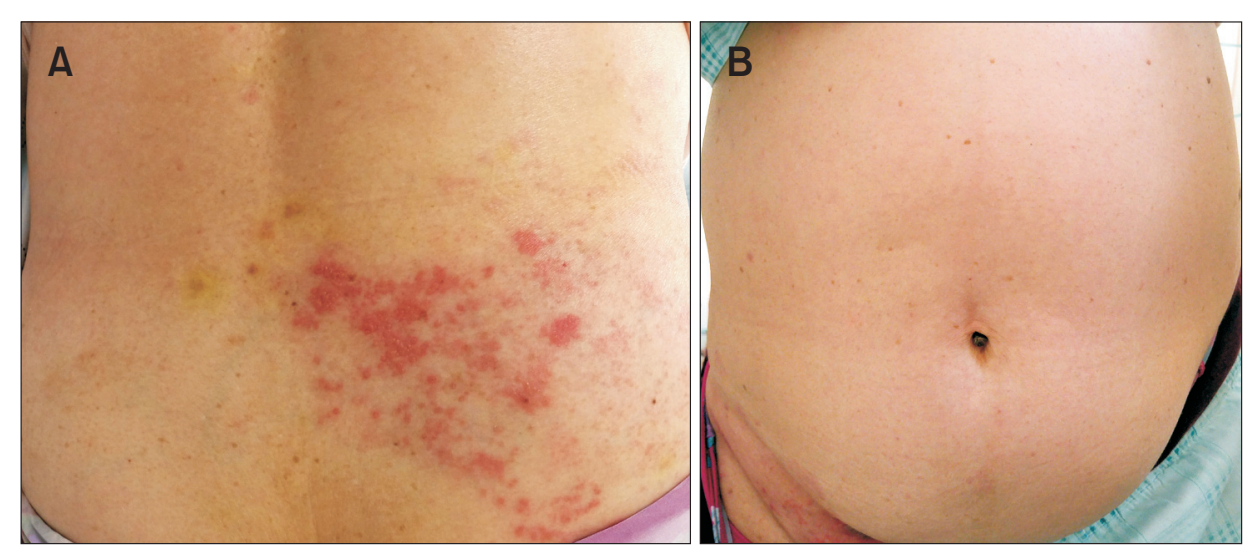

(c) 2017 The Korean Society of Neurogastroenterology and Motility
Figure 1. Physical examination. (A) Vesicular eruptions involving the right L4 dermatome and (B) marked abdominal distension were noted. 

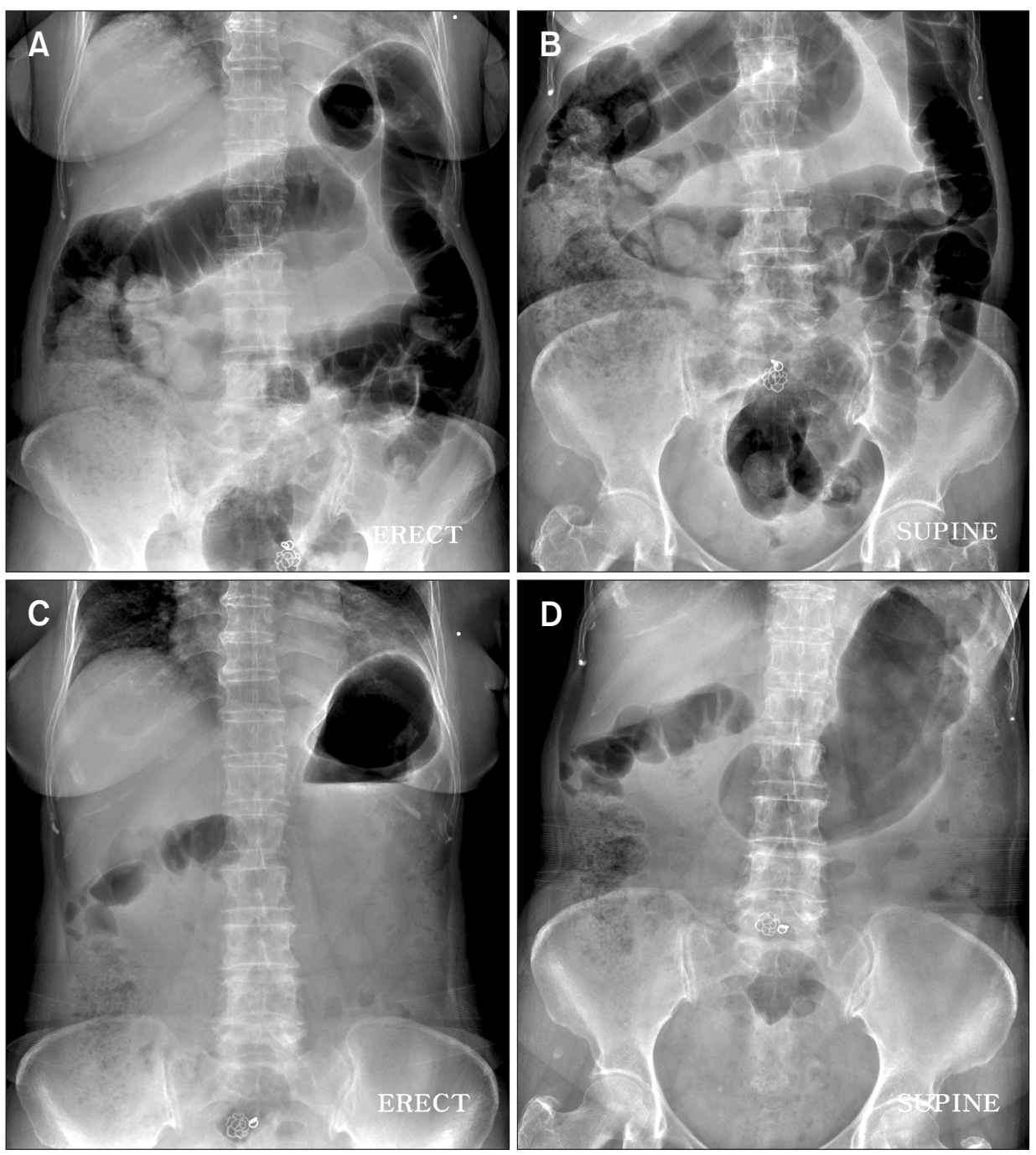

Figure 2. Radiographic findings. (A, B) Abdominal X-ray on admission shows diffuse colonic dilatation. (C, D) Colonic dilatation disappears after 10 days of conservative management.

emia or perforation occurs. Therefore, early recognition and appropriate management of unusual gastrointestinal presentations such as ACPO in patients with zoster is important to prevent potential serious outcomes.

Jae Yoon Chung, ${ }^{1}$ Jong Seol Park, ${ }^{2}$ and Yong Sung Kim ${ }^{2}$ ${ }^{1}$ Department of Anesthesiology, Wonkwang University Sanbon Hospital, Gunpo, Gyeonggi-do, Korea; and ${ }^{2}$ Department of Internal Medicine and Wonkwang Digestive Disease Research Institute, Wonkwang University Sanbon Hospital, Gunpo, Gyeonggi-do, Korea

1. Saunders MD, Kimmey MB. Systematic review: acute colonic pseudoobstruction. Aliment Pharmacol Ther 2005;22:917-925.

2. Vanek VW, Al-Salti M. Acute pseudo-obstruction of the colon (Ogilvie's syndrome). An analysis of 400 cases. Dis Colon Rectum 1986;29:203210.

3. Jellinek EH, Tulloch WS. Herpes zoster with dysfunction of bladder and anus. Lancet 1976;2:1219-1222.

4. Chernev I, Dado DN. Segmental zoster abdominal paresis/paralysis, zos- ter pseudohernia or zoster lumbar hernia? Hernia 2014;18:145-146.

5. Pui JC, Furth EE, Minda J, Montone KT. Demonstration of varicellazoster virus infection in the muscularis propria and myenteric plexi of the colon in an HIV-positive patient with herpes zoster and small bowel pseudo-obstruction (Ogilvie's syndrome). Am J Gastroenterol 2001;96:16271630.

6. Edelman DA, Antaki F, Basson MD, Salwen WA, Gruber SA, Losanoff JE. Ogilvie syndrome and herpes zoster: case report and review of the literature. J Emerg Med 2010;39:696-700.

Financial support: This work was supported by Wonkwang University 2017 (Jae Yoon Chung).

\section{Conflicts of interest: None.}

Author contributions: Concept: Jae Yoon Chung and Yong Sung Kim; data collection and/or processing: Jae Yoon Chung and Jong Seol Park; analysis and/or interpretation, literature search, and writing: Jae Yoon Chung, Jong Seol Park, and Yong Sung Kim; and critical review: Yong Sung Kim. 